Bessa MM, Lima LS, Silva MDM \& Freitas RJM (2020). Gloves as a preventive measure for Covid-19: who should use it? Research, Society and Development, 9(8): 1-10, e746974846.

\title{
Luvas como medida preventiva à Covid-19: quem deve utilizar?
}

Gloves as a preventive measure for Covid-19: who should use it?

Guantes como medida preventiva para Covid-19: ¿quién debería usarlo?

Recebido: 23/05/2020 | Revisado: 26/05/2020 | Aceito: 27/05/2020 | Publicado: 09/06/2020

Marcelino Maia Bessa

ORCID: https://orcid.org/0000-0001-6699-5109

Universidade do Estado do Rio Grande do Norte, Brasil

E-mail: marcelino.maia.18@outlook.com

Layane da Silva Lima

ORCID: https://orcid.org/0000-0002-9137-6673

Universidade do Estado do Rio Grande do Norte, Brasil

E-mail: laypb@hotmail.com

Marlison Diego Melo da Silva

ORCID: https://orcid.org/0000-0001-5541-4807

Universidade Federal Rural do Semiárido, Brasil

E-mail: marlisondiego3@gmail.com

Rodrigo Jácob Moreira de Freitas

ORCID: https://orcid.org/0000-0002-5528-2995

Universidade do Estado do Rio Grande do Norte, Brasil

E-mail: rodrigojmf@gmail.com

\section{Resumo}

Introdução: O vírus SARS-COV-2 causa a doença conhecida como infecção por coronavírus ou COVID-19. Medidas preventivas para não infecção devem ser adotadas, dentre elas, notase o uso de luvas pelos profissionais da saúde e população em geral. Objetivo: discutir sobre o uso de luvas pela pelos profissionais da saúde e pela população em geral. Metodologia: tratase de um estudo descritivo-exploratório, do tipo artigo informativo, baseado na literatura cientifica, a busca foi feita entre os dias de 15 a 23 de Maio, nas bibliotecas Biblioteca Virtual em Saúde (BVS) e Scielo, bem como em documentos da Organização Mundial da Saúde e do Ministério da Saúde. Conduzida sem filtro de tempo. Resultados: o uso de luvas é uma 
alternativa de suma importância para os profissionais de saúde, mas não indicado para o uso da população em geral. Dar ênfase que esse uso não substitui a lavagem das mãos, reforçando essa importância higiênica e que quando usadas, que se deem de forma adequada. Conclusões: Que estes profissionais façam treinamentos e atualizações de protocolos de lavagem das mãos e uso das luvas. Que a população seja desencorajada ao uso, podendo este ser feito por meio das páginas oficiais e mídias sociais, bem como por intermédio direto dos profissionais da Atenção Básica.

Palavras-chave: Luvas cirúrgicas; Prevenção primária; Infecções por Coronavirus; Pessoal de saúde; População.

\section{Abstract}

Introduction: The SARS-COV-2 virus causes the disease known as coronavirus or COVID-19 infection. Preventive measures for non-infection should be adopted, among them, the use of gloves by health professionals and the population in general is noted. Objective: to discuss the use of gloves by health professionals and the general population. Methodology: this is a descriptive-exploratory study, of the informative article type, based on scientific literature, the search was made between the 15th to the 23rd of May, in the Virtual Health Library (VHL) and Scielo libraries, as well as in documents from the World Health Organization and the Ministry of Health. Conducted without a time filter. Results: the use of gloves is an extremely important alternative for health professionals, but not indicated for the use of the general population. Emphasize that this use does not replace hand washing, reinforcing this hygienic importance and that when used they give themselves in an appropriate way. Conclusions: That these professionals do training and updates on hand washing and gloves use protocols. May the population be discouraged from using it, which can be done through the official pages and social media, as well as through direct professionals of Primary Care.

Keywords: Gloves surgical; Primary prevention; Coronavirus infections; Health personnel; Population.

\section{Resumen}

Introducción: El virus SARS-COV-2 causa la enfermedad conocida como coronavirus o infección por COVID-19. Deben adoptarse medidas preventivas para la no infección, entre ellas, el uso de guantes por parte de los profesionales de la salud y la población en general. Objetivo: discutir el uso de guantes por parte de los profesionales de la salud y la población en general. Metodología: este es un estudio descriptivo-exploratorio, del tipo de artículo 
informativo, basado en literatura científica, la búsqueda se realizó entre el 15 y el 23 de mayo, en la Biblioteca Virtual en Salud (BVS) y las bibliotecas Scielo, así en documentos de la Organización Mundial de la Salud y el Ministerio de Salud, realizado sin filtro de tiempo. Resultados: el uso de guantes es una alternativa extremadamente importante para los profesionales de la salud, pero no está indicado para el uso de la población en general. Haga hincapié en que este uso no reemplaza el lavado de manos, lo que refuerza esta importancia higiénica y que, cuando se usan, se entregan de manera adecuada. Conclusiones: que estos profesionales realizan capacitación y actualizaciones sobre el lavado de manos y el uso de guantes. Que se desanime a la población a usarlo, lo que se puede hacer a través de las páginas oficiales y las redes sociales, así como a través de profesionales directos de Atención Primaria.

Palabras clave: Guantes quirúrgicos; Prevención primaria; Infecciones por coronavirus; Personal de salud; Población.

\section{Introdução}

A rápida e incontrolável disseminação do novo coronavírus (SARS-CoV-2) pelo mundo aliada à sua gravidade fez com que, no dia 11 de março de 2020, o Diretor-Geral da Organização Mundial da Saúde (OMS) caracterizasse a situação como pandemia. Atualmente, nota-se um esforço cada vez mais intenso por parte das organizações de saúde e do poder público no sentido de conter o avanço e disseminação do SARS-CoV-2 (WHO, 2020).

O vírus SARS-COV-2 causa a doença conhecida como infecção por coronavírus ou COVID-19, caracterizada por provocar infecções respiratórias e intestinais em humanos e animais. A maioria das infecções por coronavírus em humanos são causadas por espécies de baixa patogenicidade, levando ao desenvolvimento de sintomas do resfriado comum, no entanto, podem eventualmente levar a infecções graves em grupos de risco (Zhu et al., 2020).

A disseminação pessoa-pessoa ocorre principalmente por meio de gotículas respiratórias produzidas quando alguém infectado tosse ou espirra, semelhante à maneira como a influenza e outros patógenos respiratórios se espalham (Brasil, 2020). No momento do espirro as gotículas com o vírus podem atingir pessoas que estão próximas, bem como ficam depositados sobre superfícies podendo estas tornarem-se fonte de contaminação, assim pode ocorrer com que esses microrganismo venham a entrar em contato com o nariz, boca ou olhos, com o uso das mãos (Li et al., 2020).

A precaução de gotículas e contato é recomendada para evitar a disseminação de 
COVID-19. Dessa forma, essa situação demanda medidas de prevenção, dentre elas percebese o uso de luvas por profissionais da saúde e pela população em geral (Brasil, 2020; Carvalho, 2020). A literatura traz a importância desse uso para os profissionais de saúde, porém que o uso desnecessário de luvas em situações não recomendadas representa um desperdício de recursos sem que, necessariamente, leve à redução de infecção/transmissão de agentes patogênicos (Leung, Lam \& Cheng, 2020; WHO, 2009).

Nessa perspectiva, questiona-se: como deve ser tratado o uso de luvas no que tange os profissionais de saúde e a população em geral? Essa pesquisa justifica-se para se ter uma visão mais ampliada sobre o uso de luvas, bem como uma forma de trazer informações para a população sobre o não uso ou uso adequado na situação atual. Nessa perspectiva, este estudo tem como objetivo discutir sobre o uso de luvas pela pelos profissionais da saúde e pela população em geral.

\section{Metodologia}

Trata-se de um estudo descritivo-exploratório, do tipo artigo informativo, baseado na literatura cientifica, a busca foi feita entre os dias de 15 a 23 de Maio, nas bibliotecas Biblioteca Virtual em Saúde (BVS) e Scielo, utilizando os descritores "Luvas Cirúrgicas", "Prevenção Primária", "Infecções por Coronavirus", "Pessoal de Saúde”, "População”, a qual foi conduzida sem filtro de tempo para melhor responder a questão de pesquisa (Pereira et al., 2018). Além disso, foi realizado busca em documentos da Organização Mundial da Saúde e do Ministério da Saúde.

\section{Resultados e Discussão}

\section{Medidas gerais de prevenção}

A elevada infectividade do SARS-CoV-2, agente etiológico da COVID-19, na ausência de imunidade prévia na população humana, bem como de vacina contra este vírus, faz com que o crescimento do número de casos seja exponencial. Nesse contexto, são indicadas intervenções visando inibir a transmissão entre humanos, desacelerar o espalhamento da doença, e consequentemente diminuir e postergar o pico de ocorrência na curva epidêmica (Anderson, Heesterbeek \& Hollingsworth, 2020).

Dentro dessa perspectiva de prevenção, o Ministério da saúde, como apresentado em 
Brasil (2020), aborda diversas formas de evitar e/ou transmitir a infecção, dentre elas podem citar-se lavar com frequência as mãos até a altura dos punhos, com água e sabão, ou então higienize com álcool em gel 70\%, ao tossir ou espirrar, cubra nariz e boca com lenço ou com o braço, e não com as mãos, evitar tocar olhos, nariz e boca com as mãos não lavadas, ao tocar, lave sempre as mãos como já indicado, manter uma distância mínima de cerca de 2 metros de qualquer pessoa tossindo ou espirrando, assim como evitar abraços, beijos e apertos de mãos. Adote um comportamento amigável sem contato físico, mas sempre com um sorriso no rosto.

Além disso, outro ponto bastante discutido como afirma Qualls et al. (2020) é a importância do distanciamento social, por sua vez, abrange o isolamento de casos, a quarentena aplicada a contatos, e a prática voluntária de não frequentar locais com aglomerações de pessoas, tornando-se medidas de suma importância contra a pandemia.

\section{Uso por profissionais de saúde}

Sendo a lavagem das mãos a principal medida de prevenção a Covid-19, entra-se em questão a utilização de luvas como um equipamento de proteção. Nessa perspectiva, a literatura traz que o uso adequado de luvas, associado à prática de higienização das mãos, é fator determinante para a proteção do profissional durante a assistência à saúde, seja à nível hospitalar e/ou na atenção básica. Não obstante a isso, de acordo com a NR 32, o uso de luvas não substitui o processo de higienização das mãos o que deve ocorrer, no mínimo, antes e depois do uso das mesmas (OMS, 2014; Brasil, 2005).

Assim como as máscaras, as luvas são equipamentos de proteção individual utilizados em serviços de atenção à saúde para o cuidado de pessoas contaminadas ou suspeitas. Esses equipamentos não substituem a higienização de mãos e, pelo contrário, podem se tornar veículos de disseminação do coronavírus, pois podem dar uma falsa sensação de segurança, fazendo com que haja o toque de superfícies e pessoas de forma descuidada, com contaminações, sendo que o mesmo ocorre com o uso da máscara facial (Brasil, 2005).

Sobre o uso de luvas, viu-se que é amplamente recomendado que usem luvas por duas razões principais: para evitar que os microrganismos que estão colonizando a pele das mãos, ou estejam presentes transitoriamente nas mãos do profissional de saúde sejam transmitidos aos pacientes e de um paciente para outro; e para reduzir o risco dos próprios profissionais de saúde adquirirem infecções dos pacientes. A eficácia do uso de luvas na prevenção de contaminação das mãos desses profissionais e na redução da transmissão de patógenos tem 
sido confirmada em diversos estudos clínicos (WHO, 2009; Silva et al., 2018).

Mas para que isso possa se concretizar, é se suma importância que o uso se dê de forma adequada. Destaca-se que o uso de luvas não substitui a necessidade de higienização das mãos, usar luvas quando puder ser prevista a ocorrência de contato com sangue ou outro material potencialmente infeccioso, membranas mucosas ou pele não intacta, a remoção das luvas deve ser feita após auxiliar um paciente, não se pode usar o mesmo par de luvas para assistir mais de um paciente, quando se estiver usando luvas, troca-las ou remove-las em algumas situações como: ao mudar de um sítio corporal contaminado para outro, limpo, durante o cuidado ao paciente; após tocar um local ou superfície contaminada e antes de tocar um local limpo ou o ambiente de assistência (Brasil, 2020; Brasil, 2010)

\section{População em geral}

No que tange ao uso de luvas pela população, Organização Mundial da Saúde (OMS) desaconselha essa utilização por pessoas que não estejam cuidando de pacientes com COVID19. Percebendo assim a não indicação, seja para ir aos serviços, bem como no dia-a-dia. Essa não recomendação se dá por diversas razões (Brasil, 2020).

As luvas são contaminadas tanto quanto as mãos ao entrar em contato com superfícies infectadas com o vírus. Assim, caso o indivíduo leve a mão ao rosto, o risco de contágio é o mesmo com ou sem luvas. A diferença é que as mãos podem ser frequentemente higienizadas com água e sabão ou álcool $70 \%$ - que é a principal recomendação de prevenção dada por todas as autoridades sanitárias durante a pandemia e uma forma altamente eficaz de matar o vírus -, enquanto as luvas não são lavadas (Brasil, 2020; WHO, 2009).

O Ministério da saúde diz que ainda se pode detectar contaminação por covid-19 em luvas de borracha, ou seja, se indivíduo tocar seu rosto, a contaminação vai da sua luva para o seu rosto e pode infectá-lo, ressaltando ainda que limpar regularmente as mãos oferece mais cobertura do que usar luvas de borracha (Brasil, 2020; Brasil, 2011).

Segundo as diretrizes para o uso de luvas da OMS, médicos e enfermeiros utilizam um par para cada paciente, e só as vestem em momentos específicos (como quando há a iminência de contato com fluidos corporais ou o toque em mucosas), visando não contaminar as mãos com vírus e bactérias ou transmitir algo para a pessoa (Leung, Lam \& Cheng, 2020; Silva et al., 2018).

Além disso, outro ponto importante é o fato de que, no mundo inteiro, já há escassez de itens como máscaras cirúrgicas e luvas descartáveis, algo que já tem deixado diversos 
profissionais da saúde desprotegidos, aumentando os riscos de contágio tanto para os profissionais quanto para pacientes, visto que o uso indiscriminado acarreta nesse processo. Sendo assim, o órgão é claro: só deve usar luvas quem está cuidando de alguém com COVID19 ou é um profissional da saúde (Brasil, 2020).

Um dos pontos críticos para o uso da luva e por não ser indicado é que o uso contínuo das luvas pode gerar uma contaminação cruzada. Ou seja, uma pessoa leva o vírus de um lugar para o outro sem perceber. Por isso é muito importante seguir todas as medidas de higiene e trocá-las sempre a cada ocasião. Luvas devem ser de uso único e descartadas toda vez. Destaca-se ainda, mesmo antes da pandemia, na área da saúde já há um debate sobre o uso indiscriminado de luvas, já que você protege a sua mão, mas pode levar microrganismos para outras pessoas e até para pacientes, não sendo diferente para a população em geral. Não obstante a isso, o uso de luvas sem respeitar regras de higiene não serve de nada, mas pode ser agravante (WHO, 2009; Silva et al., 2018).

Ademais, ressalta-se assim também que o uso de luvas pode resultar em oportunidades perdidas para a higiene das mãos e que o uso de luvas contaminadas pelo armazenamento incorreto e pelos momentos e técnicas inapropriados de calçamento e remoção pode resultar na transmissão de microrganismos. Não obstante a isso, é o que vem sendo amplamente discutido pelo não uso de luvas pela população (Brasil, 2020; WHO, 2009).

\section{Considerações Finais}

Os objetivos foram alcançados ao se discutir o uso de luvas pelos profissionais de saúde e pela população em geral como forma de prevenção à Covid-19. Nesse contexto, ressalta-se a importância de reconhecer a necessidade e obrigatoriedade do uso pelos profissionais e da não recomendação de uso para a população. Ressaltando-se ainda que o uso de luvas em nenhuma circunstância substitui a lavagem das mãos, podendo ser notado ainda a maior eficiência da lavagem.

No que tange aos profissionais de saúde, é necessário que esses façam a lavagem das mãos constantemente, bem como o uso da luva seja utilizado de forma consciente no intuito de diminuir a proliferação do coronavírus, prezando assim para a segurança do paciente e a si mesmo. Dessa forma, ressalta-se a importância do treinamento das instituições e dos profissionais frente a pandemia, podendo também ser realizado atualizações sobre o protocolo de lavagem das mãos, bem como o uso adequado das luvas.

Com relação a população em geral, não é indicado que seja feito o uso de luvas, uma 
vez que este pode ser entendido como uma substituição da lavagem das mãos, colocando assim em risco a si mesmo e aos demais, além de não ser uma forma de prevenção para com esse público, mas ressaltar a importância e eficácia dessa lavagem.

É necessário que os órgãos de saúde possam atuar diretamente na orientação a essa população como meio de desencorajar esse uso, mostrando o que pode ser acarretado por esse má utilização. Podem ser feita dispondo de meios oficiais de comunicação virtual como sites oficiais e mídias sociais para a diminuição e/ou sanção desse problema, bem como inserir o papel educativo dos profissionais de saúde, das entidades de saúde podendo ressaltar a Atenção Básica, por ter uma conexão de informações maior de informações com a população.

\section{Referências}

Anderson RM, Heesterbeek H \& Hollingsworth TD (2020). How will country-based mitigation measures influence the course of the Covid-19 epidemic? Lancet [Internet]. [cited 2020 Mai 22];395(10228):931-4. Available from: Available from:

https://doi.org/10.1016/S0140-6736(20)30567-5

Brasil (2005). Ministério do Trabalho e Emprego. Portaria no 485, de 11 de novembro de 2005. Aprova a norma regulamentadora $\mathrm{n}^{\circ} 32$ (Segurança e saúde no trabalho em estabelecimentos de saúde) [Internet]. Diário Oficial da República Federativa do Brasil, Brasília, DF: 2005[cited 2020 Mai 23]. Available from: http://sbbq.iq.usp.br/arquivos/seguranca/portaria485.pdf

Brasil. (2010). Agência Nacional de Vigilancia Sanitária. Primeiro desafio mundial para a segurança do paciente. Uso de luvas (técnico). Folha informativa 6: p2. Disponível em: http://www.anvisa.gov.br/servicosaude/controle/higienizacao_oms/folha\%20informativa\%20 6.pdf

Brasil. (2011). Agência Nacional de Vigilância Sanitária. Resolução no 55, de 4 de novembro de 2011. Estabelece os requisitos mínimos de identidade e qualidade para as luvas cirúrgicas e luvas para procedimentos não cirúrgicos de borracha natural, de borracha sintética, de mistura de borrachas natural e sintética e de policloreto de vinila, sob regime de vigilância sanitária. Disponível em: 
http://www.lex.com.br/doc_22237184_RESOLUCAO_N_55_DE_4_DE_NOVEMBRO_DE_ 2011.aspx

Brasil. (2020). Ministério da Saúde (BR). Protocolo de Tratamento do Novo Coronavírus (2019-nCoV). Tiragem: 1ª edição - 2020 - publicação eletrônica. Disponível em: https://portalarquivos2.saude.gov.br/images/pdf/2020/fevereiro/05/Protocolo-de-manejoclinico-para-o-novo-coronavirus-2019-ncov.pdf

Carvalho P (2020). Coronavirus, afinal vale a pena usar luva ou não ao sair de casa? Disponível em: https://www.uol.com.br/vivabem/noticias/redacao/2020/04/08/ coronavirus-afinal-devo-usar-luva-ou-nao-enfermeira-explica-se-ha-riscos.htm. Acesso em: 22mai. 2020.

Leung CC, Lam TH \& Cheng KK (2020). Mass masking in the COVID-19 epidemic: people need guidance. Lancet [Internet]. [cited 22mai 2020]; 395(10228):22-45. Available from: https://doi.org/10.1016/S0140-6736(20)30520-1

Li Q et al (2020) Early transmission dynamics in Wuhan, China, of novel coronavirusinfected pneumonia. N Engl J Med [Internet]. [cited 22mai 2020]; 382:1199- 1207. Available from: https://doi.org/10.1056/NEJMoa2001316

Organização Mundial da Saúde-OMS. (2014) SALVE VIDAS: Higienize suas Mãos. Higiene das Mãos na Assistência à Saúde Extra-hospitalar e Domiciliar e nas Instituições de Longa Permanência - Um Guia para a Implementação da Estratégia Multimodal da OMS para a Melhoria da Higiene das Mãos e da Abordagem "Meus 5 Momentos para a Higiene das Mãos"; tradução de OPAS - Brasília: Organização Pan-Americana da Saúde; Agência Nacional de Vigilância Sanitária; 73 p.

Pereira AS et al. (2018). Metodologia da pesquisa científica. [e-book]. Santa Maria. Ed. UAB/NTE/UFSM. Disponível em: https://repositorio.ufsm.br/bitstream/handle/1/15824/Lic_ Computacao_Metodologia-Pesquisa-Cientifica.pdf?sequence=1. Acesso em: 27 mai. 2020. 
Qualls N et al (2017) Community mitigation guidelines to prevent pandemic influenza United States, 2017. MMWR Recomm Rep [Internet]. [cited 2020 Mai 23];66(1):1-32. Available from: Available from: https://doi.org/10.15585/mmwr.rr6601a1

Silva DM, Marques BM, Galhardi NM, Orlandi FS \& Figueiredo RM (2018). Hands hygiene and the use of gloves by nursing team in hemodialysis service. Rev Bras Enferm [Internet]. 8;71(4):1963-9. DOI: http://dx.doi.org/10.1590/0034-7167-2017-0476

World Health Organization. (WHO). (2020). WHO Director-General's opening remarks at the media briefing on COVID-19. [Internet] [Cited in 2020 Mai 22] Available from: https://www.who.int/dg/speeches/detail/who-director-general-s-opening-remarks-atthe-media-briefing-on-covid-19---11-march-2020.

World Health Organization. Gloves use information leaflet. (2009). Disponível em: http://www.who.int/gpsc/5may/Glove_Use_Information_Leaflet.pdf

Zhu $\mathrm{N}$ et al (2020). A novel Coronavirus from patients with pneumonia in China, 2019. N Eng J Med 2020; 1-7. doi: 10.1056/NEJMoa2001017

\title{
Porcentagem de contribuição de cada autor no manuscrito
}

\author{
Marcelino Maia Bessa - 35\% \\ Layane da Silva Lima - 20\% \\ Marlison Diego Melo da Silva - 20\% \\ Rodrigo Jácob Moreira de Freitas - 25\%
}

